\title{
Research on Handoff Delay and Mobility Management Cost of Mobility Protocols in Wireless Sensor Networks
}

\author{
A. Q. Zhao and Y. Hu \\ School of Computer and Information Technology, Beijing Jiaotong University, Beijing 100044, China \\ Correspondence should be addressed to A. Q. Zhao; aqzhao@bjtu.edu.cn
}

Received 25 November 2014; Revised 18 February 2015; Accepted 4 March 2015

Academic Editor: Fei Yu

Copyright ( 2015 A. Q. Zhao and Y. Hu. This is an open access article distributed under the Creative Commons Attribution License, which permits unrestricted use, distribution, and reproduction in any medium, provided the original work is properly cited.

\begin{abstract}
An appropriate network model and some suitable performance evaluation criterions including handoff delay and mobility management cost were proposed in this paper. And in this base the performance of Mobile IP protocol and various micromobility protocols was comprehensively compared and investigated. The research results show that the performance is mainly influenced by two factors which are route update methods of mobility support protocols and mobile network parameters. The route update time and mobility management cost of micromobility protocol are obviously shorter than that of Mobile IP. In all researched micromobility protocols, the route update method of Mobile IP Regional Registration protocol has the optimal performance.
\end{abstract}

\section{Introduction}

Mobile IP protocol [1-3] is the most basic mobile support protocol of the Internet. With the development of network and application, Mobile IP is increasingly exposed to serious performance defects. Therefore, micromobility protocols were proposed, such as micromobility protocols, Mobile IP Regional Registration (MIP-RR) protocol [4, 5], Cellular IP protocol (CIP) [6, 7], and HAWAII protocol $[8,9]$.

Handoff is the most basic operation of the mobile network and the most important content of mobility support protocol research. The handoff performance has a crucial impact on the performance of the mobile network [10-13]. Network layer handoff delay is the most important indicator to measure mobility support protocols, and other handoff performance indicators, for example, packet loss and application throughput decline when making handoff, are all relate to handoff delay. This paper focused on performance evaluation of Mobile IP and a variety of micromobility protocol network layer handoff delay.

To ensure mobility support protocols' use in practice, we believe that the other performance indicator, that is, mobility management cost, is as important as handoff performance. Mobility management cost of mobility support was studied in [14-17]. Paper [14] studied theoretically the influence that the packets arrival intervals have on the mobility management cost of micromobility protocols and pointed out that the factors affecting its performance is the average packet arrival intervals, rather than the type of interval time distribution. Paper [15] compared the management cost of Mobile IP and micromobility protocols mainly through simulation method.

In the mobility support protocols handoff performance study, we put forward an idea. Firstly a suitable network model was proposed, analyze the factors affecting handoff performance on the basis of the network model, and theoretically compare various mobility support protocols' handoff performance. This paper focused on the research of mobility management cost of mobility support protocols and made analysis and comparison of mobility management cost among various mobility support protocols.

\section{Network Modeling}

The wireless access network is constituted by several administration domains [13]. And every administration domain is connected to the Internet through GW (Gateway). The cellular model adopted in the administration domain is shown in Figure 1. It is a concentric circle model (the usually used hexagonal model is not adopted in this paper for calculating 


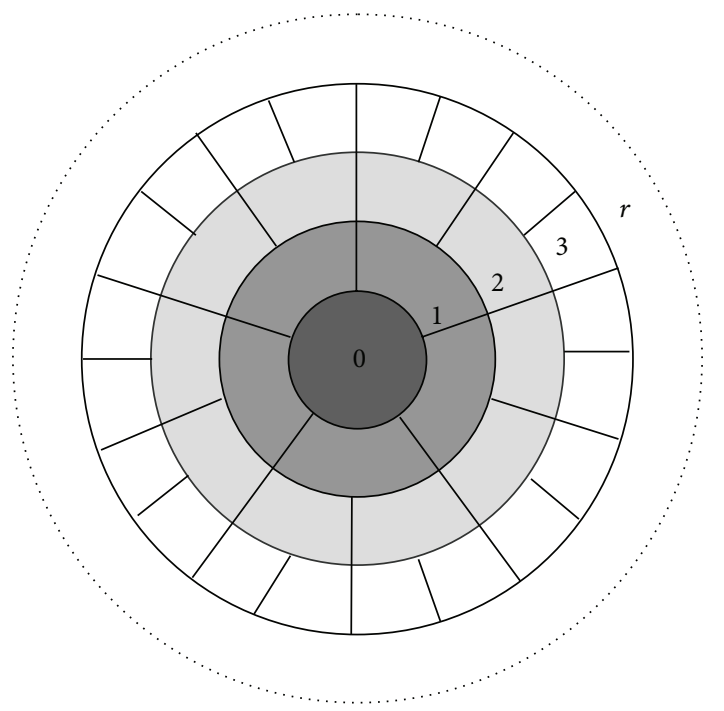

Figure 1: Cellular model of the administration domain.

simplification). The model has a center cell (defined as the cell in layer 0 ). Other cells are distributed around the center cell with their layer number of $1,2,3$, and so on. There are 5 cells in layer 1 . The number of the cell in layer $i+1(i=1,2,3, \ldots)$ is two times more than the number of the cell in layer $i$. Each cell has five adjacent cells. The cell in layer 0 adjoins 5 cells in layer 1 , while the cell in layer $i(i=1,2,3, \ldots)$ adjoins 1 cell in layer $i-1,2$ cells in layer $i$, and 2 cells in layer $i+1$. A BS (Base Station) which has the function of MRA (Mobile Routing Agent) [18] is set in each cell. We make the BS in layer 0 Gateway of the whole administration domain. All adjacent BSs are linked by wired links. And the distance between them is 1 hop.

Suppose the radius of administration domain is $r$; the MHs are uniformly distributed in the cells in administration domains. We denote the probability that the $\mathrm{MH}$ occurs in a cell in layer $i$ by $p_{i}$. So $p_{i}$ is given by

$$
p_{i}=\frac{N_{i}}{N}=\frac{N_{i}}{\sum_{i=0}^{r} N_{i}}= \begin{cases}\frac{1}{\left(5 * 2^{r}-4\right)} & i=0 \\ 5 * \frac{2^{i-1}}{\left(5 * 2^{r}-4\right)} & i=1,2, \ldots, r .\end{cases}
$$

In the formula, $N_{i}$ and $N$ denote the number of the cell in layer $i$ and the number of the cell in the whole administration domain, respectively.

Suppose the distance from a $i$-layer cell to GW is $i$; then the average distance between $\mathrm{MH}$-cells and GW is given by

$$
d=\sum_{i=0}^{r} i * p_{i}=\frac{5\left[(r-1) 2^{r}+1\right]}{\left(5 * 2^{r}-4\right)} .
$$

Suppose the probabilities in which a MH executes handoffs to its five adjacent cells are equal. The probability that the MH executes a handoff from layer $i(i=1,2,3, \ldots, r)$ to layer $j$ is

$$
p_{i, j}= \begin{cases}\frac{1}{5} & j=i-1 \\ \frac{2}{5} & j=i \\ \frac{2}{5} & j=i+1 .\end{cases}
$$

MH uses announcement packets that are broadcasted by BS periodically to trigger network layer handoff; the handoff process is as follows: before the handoff, $\mathrm{MH}$ sends and receives data through the old BS. When $\mathrm{MH}$ detected the need to handoff, it disconnects the connection with the old BS at first and then establishes a connection with the new BS. $\mathrm{MH}$ sends the route update message to update the location information of itself when it receives the first announcement packet from the new BS. After the completion of the route update, $\mathrm{MH}$ can send and receive data through the new BS.

\section{Handoff Delay}

Handoff process shows that the network layer handoff delay consists of two parts: one is the elapsed time from $\mathrm{MH}$ disconnecting the connection with the old BS to $\mathrm{MH}$ receiving the first announcement packet from the new BS, called movement detection time. The second is the time of $\mathrm{MH}$ sending the route update message to update the location information of itself, called route update time. Movement detection time is related to link layer handoff time and the cycle of BS broadcast announcement packets, rather than the mobility support protocols. However, route update time we will focus on hereinafter is determined by route update methods of mobility support protocols.

Route update time consists of two parts. One is the route update packet transmission delay. The other is the route update packet processing delay of mobile support node. Suppose, in the administration domain, the transmission delay of the route update packets per unit distance is $T$; the total one-way transmission delay of route update packets in the Internet is $w * T$; processing delay per route update packet of mobile support node is $p * T$. We first compared the route update time of Mobile IP and that of micromobility protocols and then the route update time of different micromobility protocols.

3.1. Comparison of Mobile IP with Micromobility Protocols. In the case of using Mobile IP, route update packets from $\mathrm{MH}$ were passed to the new BS and then the GW, arriving at the local agent at last. The reply packets along the opposite path return to $\mathrm{MH}$. We denote route update time by $T_{\mathrm{MIP}}$. $T_{\mathrm{MIP}}$ is

$$
T_{\text {MIP }}=\left[2\left(d^{\prime}+1\right)+2 w+3 p\right] T .
$$

In the formula above, $d^{\prime}$ denotes the average distance between new BS and GW; from formula (2) and (3), we could get $d^{\prime}$ which is

$$
d^{\prime}=\sum_{i, j}(d+j-i) * p_{i, j}=d+\frac{1}{5} .
$$




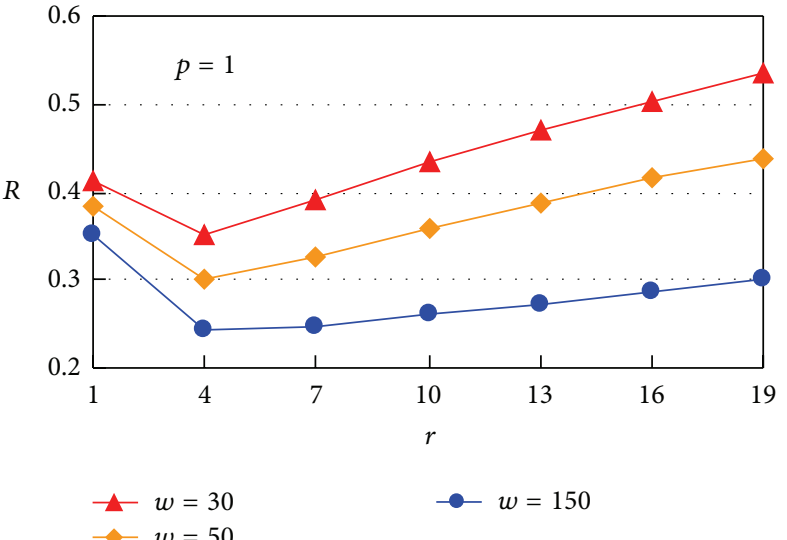

(a)

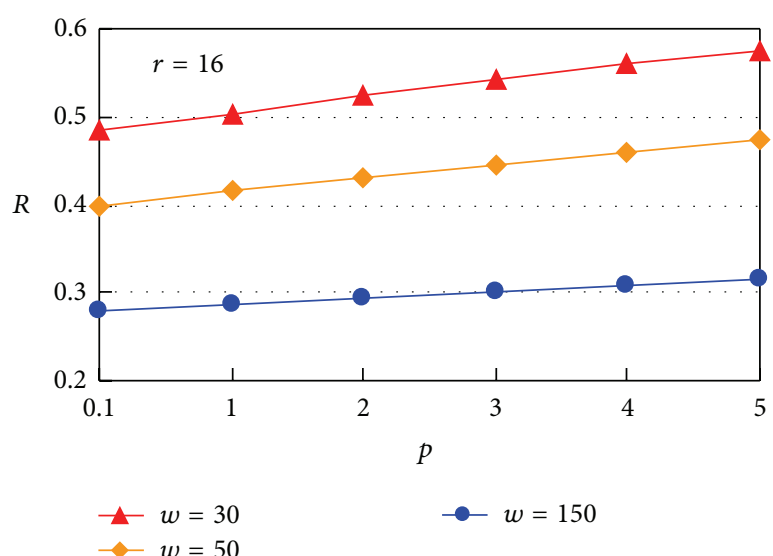

(b)

FIGURE 2: Performance of Mobile IP and micromobility protocols.

In the case of using micromobility protocols (with twolayer mobile as an example), route update methods are different between interdomain handoff and intradomain handoff. Interdomain handoff is processed just as that of Mobile IP; route update time $T_{\text {inter }}$ is

$$
T_{\text {inter }}=[2(r+1)+2 w+5 p] T .
$$

Micromobility protocols were used to process intradomain handoff. The route update packets from $\mathrm{MH}$ were passed to the new BS and then arrive at GW. The reply packets along the opposite path return to $\mathrm{MH}$. We denote route update time by $T_{\text {intra }} \cdot T_{\text {intra }}$ is

$$
T_{\text {intra }}=\left[2\left(d^{\prime}+1\right)+3 p\right] T .
$$

Interdomain handoff occurs only when the MHs are in the administration domain boundary cells. According to formula (1) and (3), the probability of $\mathrm{MH}$ interdomain handoff is

$$
p_{\text {inter }}=p_{r} * p_{r, r+1}=\frac{2^{r}}{\left(5 * 2^{r}-4\right)} .
$$

We got the average route update time of micromobility protocols from formula (6), (7), and (8):

$$
T_{\text {MMP }}=p_{\text {inter }} * T_{\text {inter }}+\left(1-p_{\text {inter }}\right) * T_{\text {intra }} .
$$

To compare the route update time of Mobile IP protocol and micromobility protocols, we calculated the ratio of the two as follows:

$$
R=\frac{T_{\mathrm{MMP}}}{T_{\mathrm{MIP}}} .
$$

We take $T=1$; when $w$ takes different values, ratio $R$ of the route update time was calculated with the change of $p$ and the value of the administration domain radius $r$. The result is shown in Figure 2.
Figure 2 shows that $R<1$ in any case; this means that the introduction of micromobility protocols is necessary because the average route update time of micromobility protocol is obviously shorter than that of Mobile IP. When $r$ is small, $R$ is at a middle level; as $r$ increases, $R$ decreases rapidly and soon reaches the minimum; then, as the further increases of $r, R$ gradually increases slowly. The change trend above indicates that the advantage of micromobility protocols is related to the size of the administration domain; there is an optimal $r$ which can be used as one of the considerations to determine administration domain size. Figure 2 also shows that $R$ declines as $w$ increases. For the larger $w$ is, the farther away $\mathrm{MH}$ from the local network is, so the farther away $\mathrm{MH}$ from the local network, the more obvious advantages of micromobility protocols. The introduction of micromobility protocols result to the nodes that process route update packets increases, so, with $p$ increasing, the advantage of the introduction of micromobility protocols decreases.

3.2. Comparison of Different Micromobility Protocols. We compared the route update time of MIP-RR, CIP, and HAWAII protocol only in situation of intradomain handoff, for micromobility protocols all adopt Mobile IP protocol to process interdomain handoff. Suppose $\mathrm{MH}$ is in an $i$ th layer cell of the administration domain before handoff $(i=$ $1,2, \ldots, r-1)$; the packet forwarding path of $\mathrm{MH}$ within domain is the optimal path from GW to the current BS.

In the case of MIP-RR protocol, route update packets from $\mathrm{MH}$ were sent to $\mathrm{GW}$ via new $\mathrm{BS}$ when handoff happens, ending at cross MRA (the lowest level of public MRA on both new route and old route of $\mathrm{MH}$ ) [6]. The reply packets along the opposite path return to $\mathrm{MH}$. If $\mathrm{MH}$ handoff is from the $i$ th layer to the $(i-1)$ th layer or the $(i+1)$ th layer, cross MRA will be the new BS or the old BS, respectively. Their route update times are calculated as follows:

$$
\begin{aligned}
T_{\text {MIP-RR }(i, i-1)} & =(2+p) T, \\
T_{\text {MIP-RR }(i, i+1)} & =(4+3 p) T .
\end{aligned}
$$




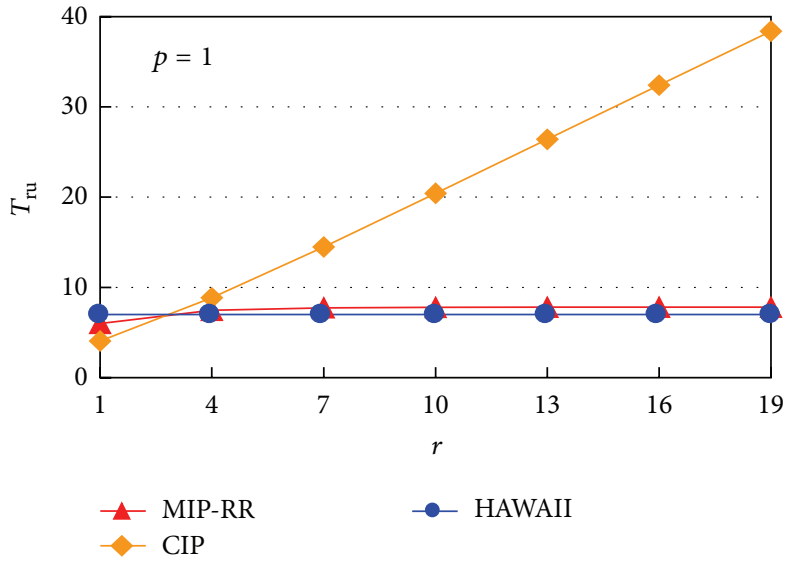

(a)

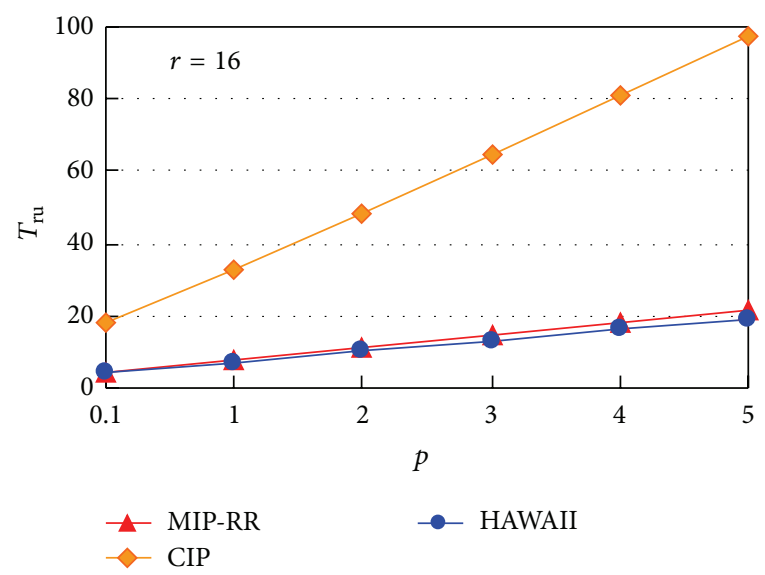

(b)

FIGURE 3: Performance of different micromobility protocols.

If $\mathrm{MH}$ handoff happens within the ith layer, assume that cross MRA is a BS at the $j$ th layer, and then route update time is

$$
T_{\mathrm{MIP-RR}(i, i) j}=[2(i-j+1)+(2(i-j)+1) p] T .
$$

The probability of cross MRA in the jth layer cell can be obtained by the administration domain structure as follows:

$$
p_{(i, i) j}= \begin{cases}\frac{1}{2^{i-1}} & j=0 \\ \frac{1}{2^{i-j}} & j=1,2, \ldots, i-1 .\end{cases}
$$

Then we got the route update time in case of MH handoff happens within the $i$ th layer which is

$$
\begin{aligned}
T_{\mathrm{MIP}-\mathrm{RR}(i, i)} & =\sum_{j=0}^{i-1} T_{\mathrm{MIP}-\mathrm{RR}(i, i) j} * p_{(i, i) j} \\
& =\left[6-\frac{4}{2^{i}}+\left(5-\frac{4}{2^{i}}\right) p\right] T .
\end{aligned}
$$

According to formula (11), (14), and (3), we could get the route update time when $\mathrm{MH}$ is in the $i$ th layer cell of the administration domain:

$$
\begin{aligned}
T_{\mathrm{MIP}-\mathrm{RR}(i)} & =\sum_{j} p_{i, j} * T_{\mathrm{MIP}-\mathrm{RR}(i, j)} \\
& =\left[\frac{22}{5}-\frac{8}{\left(5 * 2^{i}\right)}+\left(\frac{17}{5}-\frac{8}{\left(5 * 2^{i}\right)}\right) p\right] T .
\end{aligned}
$$

In the case of CIP protocol, route update packets from $\mathrm{MH}$ were sent to GW via new BS when handoff happens but reply packets are not need, so route update time is only related to the distance between new BS and GW. Route update time when $\mathrm{MH}$ handoff happens from the $i$ th layer cell to the $j$ th layer $(j=i-1, i, i+1)$ cell is as follows:

$$
T_{\mathrm{CIP}(i, j)}=(j+1)(1+p) T .
$$

Similarly, the route update time of CIP protocol when $\mathrm{MH}$ is in the $i$ th layer cell of the administration domain is

$$
T_{\mathrm{CIP}(i)}=\sum_{j} p_{i, j} * T_{\mathrm{CIP}(i, j)}=\left(i+\frac{6}{5}\right)(1+p) T .
$$

In the case of HAWAII protocol, route update packets from $\mathrm{MH}$ were sent to the old BS via new BS when handoff happens and the reply packets along the opposite path return to $\mathrm{MH}$. Because the old BS and the new BS are always directly connected to each other in all network models, route update time of HAWAII protocol when handoff happens has nothing to do with MH's location:

$$
T_{\text {HAWAII }}=(4+3 p) T .
$$

As to formula (15) and (17), if we replace $i$ with $d$ which is the average distance from the cell that MH locates to GW, we would get the average update time of MIP-RR protocol and CIP protocol. We take $T=1$; the average route update time values $\left(T_{\mathrm{ru}}\right)$ of different micromobility protocols when $r$ and $p$ take different values, respectively, are shown in Figure 3.

Figure 3 shows that, with the increase of $r$, the CIP protocol route update time increases sharply; MIP-RR protocol route update time slowly increases and converges to a fixed value while HAWAII protocol path update time remains the same. With the increase of $p$, every micromobility protocol route update time shows a linear growth. The slope of CIP protocol is greater and increases with the increase of $r$ while the slope of MIP-RR protocol and HAWAII protocol is smaller and is less affected or not affected by $r$.

CIP protocol route update time is much larger than that of MIP-RR protocol and HAWAII protocol in most cases; this is because CIP protocol route update packets need to be sent to GW continuously, so this route update method is not advisable. Since we used the adjacent BS, all interconnect structure network model, making HAWAII protocol work in the best environment, so HAWAII protocol route update time is shortest in Figure 3, but for other network structure (such as strict tree structure) HAWAII protocol route update 
time will increase. From Figure 3, we observed that MIP-RR protocol route update time is very close to the optimal value of that of HAWAII protocol and has a clear upper bound, which is a very nice feature that can ensure the maximum delay of handoff. Therefore the route update method of MIP$\mathrm{RR}$ protocol has the best performance.

\section{Mobility Management Cost}

Broadly speaking, mobility management cost refers to all of the costs of supporting MH mobility, including the terminals and mobility support nodes processing costs and the bandwidth cost of the network and position database storage overhead. This paper mainly focused on the signaling overhead brought to network in order to support MHs' mobility which is an important performance indicator when measuring mobility support protocols' performance.

In the sections below, we compared the signaling overhead when only using Mobile IP with introducing hierarchical mobility and when applying different micromobility protocols. First we present the following definition.

(1) The signaling overhead of route update packets transmission in administration domain cable link in bytes equals the value of packet size multiply link distance in hop count.

(2) Because of the limit of wireless link bandwidth resources, the signaling overhead of route update packets transmission in the wireless link is $\alpha$ times more than that of cable link.

(3) Assume the distance from GW to the HA of MH is $w$ hop and the signaling overhead of route update packets transmission in the wireless link is $\beta$ times more than that of cable link, for WAN bandwidth is expensive, too.

(4) Denote the handoff frequency of $\mathrm{MH}$ by $F_{\mathrm{HO}}$.

4.1. Comparison of Mobile IP with Micromobility Protocols. In the case of using Mobile IP, route update packets from $\mathrm{MH}$ were passed to HA through GW; the reply packets along the opposite path return to MH. In order to maintain path information, $\mathrm{MH}$ need periodically to send refresh message to HA (use route update packet to refresh.). We denote refresh message sending frequency by $F_{\mathrm{RN}}$ and the size of route update packet and reply packet by $R_{\text {update }}$ and $R_{\text {reply, }}$, respectively. The signaling overhead brought to network in order to support MHs' mobility using Mobile IP is

$$
C_{\mathrm{MIP}}=\left(\alpha+D^{\prime}+\beta w\right)\left(R_{\text {update }}+R_{\text {reply }}\right)\left(F_{\mathrm{HO}}+F_{\mathrm{RN}}\right) .
$$

In the formula above, $D^{\prime}$ denotes the average distance between new BS and GW; from formula (2) and (3), we could get $D^{\prime}$ :

$$
D^{\prime}=\sum_{i, j}(D+j-i) * p_{i, j}=D+\frac{1}{5}
$$

In the case of introducing hierarchical mobility, route update packets from $\mathrm{MH}$ were passed to HA when handoff happens, and the signaling overhead brought to network is the same as that of using Mobile IP. As to intradomain handoff, in the worst cases, path update packets from $\mathrm{MH}$ are sent to the administration domain GW and reply packets along the opposite path are to return to $\mathrm{MH}$. In order to maintain path information, $\mathrm{MH}$ need periodically to send refresh message to HA. The signaling overhead brought to network when interdomain handoff and intradomain handoff happen once can be formulated as follows, respectively:

$$
\begin{gathered}
C_{\text {inter }}=\left(\alpha+D^{\prime}+\beta w\right)\left(R_{\text {update }}+R_{\text {reply }}\right), \\
C_{\text {intra }}=\left(\alpha+D^{\prime}\right)\left(R_{\text {update }}+R_{\text {reply }}\right) .
\end{gathered}
$$

From formula (1), the probability of $\mathrm{MH}$ interdomain handoff can be calculated as follows:

$$
p_{\text {inter }}=p_{R} * p_{R, R+1}=\frac{2^{R}}{\left(5 * 2^{R}-4\right)} .
$$

So the signaling overhead brought to network in order to support MHs' mobility introducing hierarchical mobility is

$$
\begin{aligned}
C_{\mathrm{HM}}= & {\left[p_{\text {inter }} * C_{\text {inter }}+\left(1-p_{\text {inter }}\right) * C_{\text {intra }}\right] } \\
& * F_{\mathrm{HO}}+C_{\text {inter }} F_{\mathrm{RN}} \\
= & {\left[\left(\alpha+D^{\prime}+\beta w p_{\text {inter }}\right) F_{\mathrm{HO}}+\left(\alpha+D^{\prime}+\beta w\right) F_{\mathrm{RN}}\right] } \\
& \cdot\left(R_{\text {update }}+R_{\text {reply }}\right) .
\end{aligned}
$$

The difference of the signaling overhead brought to network in order to support MHs' mobility when only using Mobile IP with introducing hierarchical mobility is calculated as follows:

$$
C=C_{\mathrm{MIP}}-C_{\mathrm{HM}}=\left(1-p_{\text {inter }}\right) \beta w F_{\mathrm{HO}}\left(R_{\text {update }}+R_{\text {reply }}\right) .
$$

Because $p_{\text {inter }}<1$, so $C>0$, which means that the signaling overhead when only using Mobile IP is larger than that of introducing hierarchical mobility in any case. In order to study the advantage of introducing hierarchical mobility and micromobility protocols, we take $\beta=2, F_{\mathrm{HO}}=$ 0.25 times $/ \mathrm{s}$, and $R_{\text {update }}=R_{\text {reply }}=60$ bytes and calculated out different $w$ values and $C$ values when $R$ takes different values. The results are shown in Figure 4.

With the increase of the administration domain radius $R$, the difference of the signaling overhead brought to network in order to support MHs' mobility when only using Mobile IP with introducing hierarchical mobility $C$ also increases, but the increase rate decreases gradually, and finally the difference converges to a fixed value (under the situation of $w=10,50$, and 100 , the fixed value is 480,2400 , and 4800 , resp.). This suggests that when the administration domain is not big, the bigger the $R$ is, the more the advantages of 


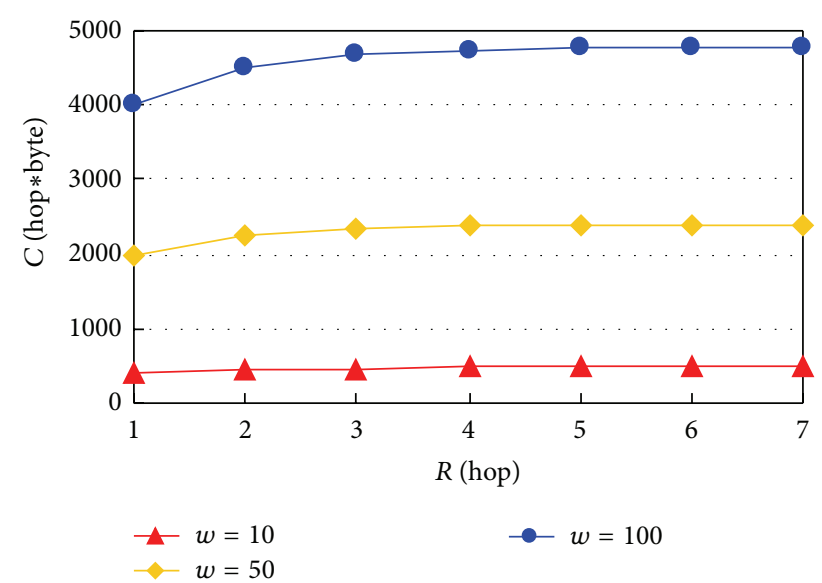

FIgURE 4: Signaling overhead of Mobile IP and micromobility protocols.

introducing hierarchical mobility are. But in the case of administration domain being very large, this advantage is not shown. In addition, as the increase of the distance between administration domain GW and HA, $C$ shows a linear growth, which suggests that the further away $\mathrm{MH}$ from the local network is, the more advantages introducing hierarchical mobility shows.

4.2. Comparison of Different Micromobility Protocols. In the case of the comparison of the signaling overhead brought to network in order to support MHs' mobility when applying different micromobility protocols such as MIP-RR, CIP, and HAWAII, because all of them adopted Mobile IP in interdomain handoff situation, we just discuss the comparison in intradomain situation.

Suppose $\mathrm{MH}$ is in an $i$ th layer cell of the administration domain before handoff $(i=1,2, \ldots, R-1)$; the packet forwarding path of $\mathrm{MH}$ within domain is the optimal path from GW to the current BS. There are three cases that may occur (Figure 5), and the handoff probability of the various situations is described by formula (3).

In the case of MIP-RR protocol, the signaling overhead brought to network in order to support MHs' mobility comprises two parts: one part is brought by Mobile IP Regional Registration request packets and reply packets which are used to establish new route. Request packets from $\mathrm{MH}$ were sent to GW via new BS and ending at cross MRA (the lowest level of public MRA on both new route and old route of $\mathrm{MH}$ ). The reply packets along the opposite path return to $\mathrm{MH}$. The other part is brought by binding update packets and reply packets which are used to delete the old route; binding update packets are sent to the old BS by the new BS; then the old BS forward the packets to GW direction and use reply packets to respond layer by layer until cross MRA; cross MRA send reply packets to $\mathrm{MH}$ at last. We denote the size of regional registration request packets and reply packets and binding update packets and reply packets by $R_{\mathrm{RR}}, R_{\mathrm{RP}}, R_{\mathrm{BU}}$, and $R_{\mathrm{BA}}$, respectively.

If $\mathrm{MH}$ handoff happens from the $i$ th layer to the $(i-1)$ th layer (Figure 5(a)), the new BS is cross MRA and the new
BS links directly to the old BS; signaling overhead brought to network when handoff happens is

$$
\begin{aligned}
C_{\mathrm{MIP}-\mathrm{RR}(i, i-1)}= & \alpha\left(R_{\mathrm{RR}}+R_{\mathrm{RP}}\right) F_{\mathrm{HO}} \\
& +\left[2 R_{\mathrm{BU}}+(\alpha+1) R_{\mathrm{BA}}\right] F_{\mathrm{HO}} .
\end{aligned}
$$

In the situation that $\mathrm{MH}$ handoff happens from the $i$ th layer to the $(i+1)$ th layer (Figure $5(\mathrm{~b}))$, the old BS is cross MRA and the new BS links directly to the old BS; signaling overhead brought to network when handoff happens is

$$
\begin{aligned}
C_{\mathrm{MIP}-\mathrm{RR}(i, i+1)}= & (\alpha+1)\left(R_{\mathrm{RR}}+R_{\mathrm{RP}}\right) F_{\mathrm{HO}} \\
& +\left[R_{\mathrm{BU}}+(\alpha+1) R_{\mathrm{BA}}\right] F_{\mathrm{HO}} .
\end{aligned}
$$

If $\mathrm{MH}$ handoff happens within the $i$ th layer, assume that cross MRA is a BS at the $j$ th layer cell $(j=i-1, i-2, \ldots, 0)$; signaling overhead brought to network when handoff happens is

$$
\begin{aligned}
C_{\mathrm{MIP}-\mathrm{RR}(i, i) j=} & (\alpha+i-j)\left(R_{\mathrm{RR}}+R_{\mathrm{RP}}\right) F_{\mathrm{HO}} \\
& +\left[(i-j+1) R_{\mathrm{BU}}+(\alpha+2(i-j)) R_{\mathrm{BA}}\right] F_{\mathrm{HO}} .
\end{aligned}
$$

The probability of cross MRA in the $j$ th layer cell can be obtained by the administration domain structure as follows:

$$
p_{(i, i) j}= \begin{cases}\frac{1}{2^{i-1}} & j=0 \\ \frac{1}{2^{i-j}} & j=1,2, \ldots, i-1 .\end{cases}
$$

According to formula (27) and (28), we could get the signaling overhead brought to network when $\mathrm{MH}$ handoff happens within the $i$ th layer:

$$
C_{\mathrm{MIP}-\mathrm{RR}(i, i)}=\sum_{j=0}^{i-1} C_{\mathrm{MIP}-\mathrm{RR}(i, i) j} * p_{(i, i) j}
$$

We got the signaling overhead brought to network when $\mathrm{MH}$ handoff happens in the situation that $\mathrm{MH}$ is located in an $i$ th layer cell of the administration domain and uses the MIP-RR protocol from formula (25), (26), (29), and (3):

$$
\begin{aligned}
C_{\mathrm{MIP}-\mathrm{RR}(i)}= & \frac{1}{5} C_{\mathrm{MIP}-\mathrm{RR}(i, i-1)}+\frac{2}{5} C_{\mathrm{MIP}-\mathrm{RR}(i, i+1)} \\
& +\frac{2}{5} C_{\mathrm{MIP}-\mathrm{RR}(i, i)} .
\end{aligned}
$$

MIP-RR protocol sends out route delete packets to delete the old route explicitly. But CIP protocol uses a very different route maintenance strategy; the old route would not be deleted explicitly but would be automatically deleted due to timeout; for this reason, $\mathrm{MH}$ must periodically send refresh packets to prevent the route information from being deleted automatically. As to CIP, though $\mathrm{MH}$ must send update packets and refresh packets to GW when every handoff happens, MH uses data packets instead of update packets and refresh packets to update and refresh the route when $\mathrm{MH}$ 


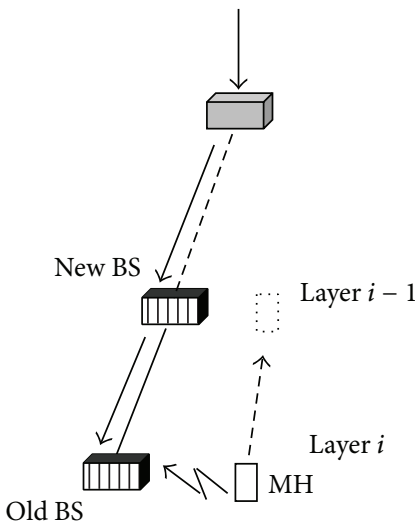

(a)

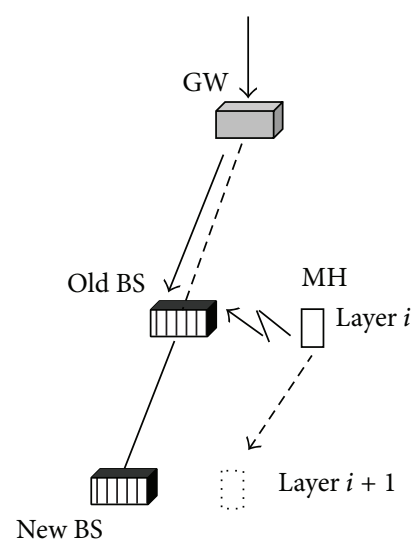

(b)

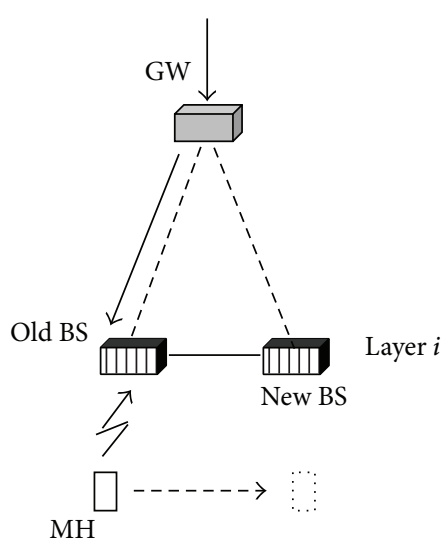

(c)

FIGURE 5: Three cases for MH handoff.

need to send out data to reduce network signaling overhead. Set the probability of the need of sending update packets and refresh packets to update and refresh the route as $p$, the size of route update packet as $R_{\mathrm{RU}}$, descending packet rate as $v$, and path information timeout time and refresh cycle ratio as $\gamma$. The literature [13] calculated the best refresh cycle as follows:

$$
T_{\mathrm{RU}}=\sqrt{\frac{p R_{\mathrm{RU}}}{\left[(\gamma-1 / 2) v F_{\mathrm{HO}}\right]}} .
$$

In the case of CIP protocol, route update packets from $\mathrm{MH}$ were sent to GW via new BS when handoff happens but reply packets are not needed, so the signaling overhead brought to network in order to support MHs' mobility is only related to the distance between new BS and GW. The signaling overheads brought to network by refreshing periodically when $\mathrm{MH}$ handoff happens from the $i$ th layer cell to the $(i-1)$ th layer, the $(i+1)$ th layer, and the $i$ th layer cell are shown as follows:

$$
\begin{gathered}
C_{\mathrm{CIP}(i, i-1)}=p(\alpha+i-1) R_{\mathrm{RU}} F_{\mathrm{HO}}, \\
C_{\mathrm{CIP}(i, i+1)}=p(\alpha+i+1) R_{\mathrm{RU}} F_{\mathrm{HO}}, \\
C_{\mathrm{CIP}(i, i)}=p(\alpha+i) R_{\mathrm{RU}} F_{\mathrm{HO}}, \\
C_{\mathrm{CIP}(i) \mathrm{Refresh}}=\frac{p(\alpha+i) R_{\mathrm{RU}}}{T_{\mathrm{RU}}} .
\end{gathered}
$$

According to formula (32) and (3), we could get the signaling overhead brought to network when $\mathrm{MH}$ handoff happens in the $i$ th layer cell of the administration domain:

$$
\begin{aligned}
C_{\mathrm{CIP}(i)}= & \frac{1}{5} C_{\mathrm{CIP}(i, i-1)}+\frac{2}{5} C_{\mathrm{CIP}(i, i+1)}+\frac{2}{5} C_{\mathrm{CIP}(i, i)} \\
& +C_{\mathrm{CIP}(i) \text { Refresh }} .
\end{aligned}
$$

In the case of HAWAII protocol, route establishing packets from MH were sent to the old BS via new BS when handoff happens and the reply packets along the opposite path return to $\mathrm{MH}$. This process completes the establishment

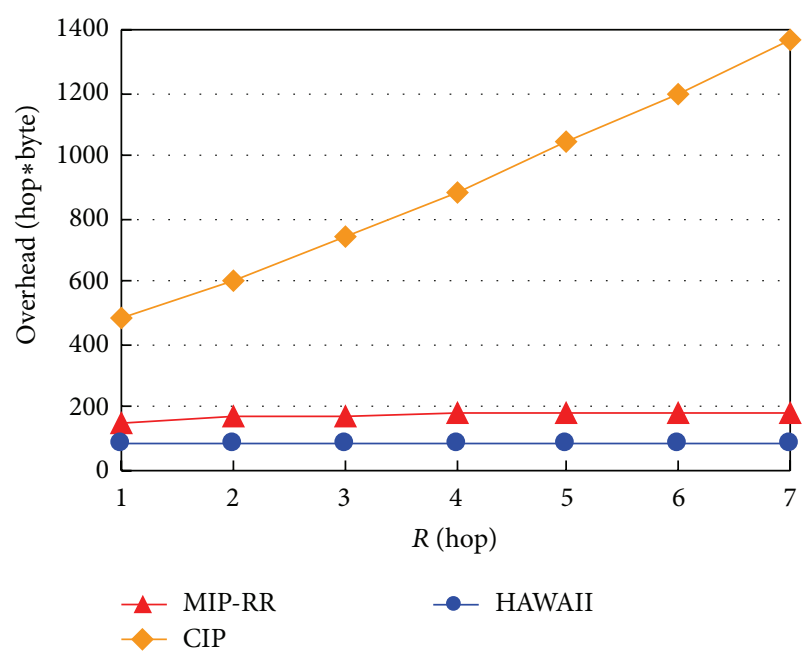

FIGURE 6: Signaling overhead of different micromobility protocols.

of the new route information and the deletion of the old route information. Set the size of route establishing packet as $R_{\mathrm{PS}}$. Because the new BS is always directly connected to the old BS, so the signaling overhead brought to network has nothing to do with the position of $\mathrm{MH}$; it is

$$
C_{\text {HAWAII }}=2(\alpha+1) R_{\mathrm{PS}} F_{\mathrm{HO}} .
$$

As to the formula above, if we replace $i$ with $D$ which is the average distance from the cell that $\mathrm{MH}$ locates to GW, and take $\alpha=2, F_{\mathrm{HO}}=0.25$ times $/ \mathrm{s}, p=0.1, \gamma=3, v=64 \mathrm{kbps}$, all the route update packets (including reply packets) have the same size of 60 bytes. We could calculate the signaling overhead brought to network in order to support MHs' mobility, respectively, with administration domain radius $R$ changing when applying various mobility support protocols. The results are shown in Figure 6.

Figure 6 shows that CIP protocol signaling overhead brought to network in order to support MHs' mobility increases with the increase of $R$, and much greater than other protocols. This phenomenon is determined by CIP protocol 
route maintenance strategy (frequently sends periodic refresh packets) and route update method (route update packets sent to GW rather than cross MRA). Though MH uses data packets instead of update packets and refresh packets to update and refresh the route when $\mathrm{MH}$ need to send out data to reduce network signaling overhead, even if $90 \%$ of the route update packets can be piggybacked by data packet ( $p=0.1$ ), the signaling overhead is also quite large (especially administration domain scope in a larger case), so CIP protocol route maintenance strategy and route update method are not desirable.

We can find that MIP-RR protocol signaling overhead brought to network in order to support MHs' mobility slightly increased with the increase of $R$ and with a clear upper bound. However HAWAII protocol signaling overhead brought to network in order to support MHs mobility is unaffected. In the case of the same administration domain size, MIPRR protocol signaling overhead is slightly larger than that of HAWAII protocol. HAWAII protocol uses the same route maintenance strategy (delete the old route explicitly) as MIPRR protocol, but MIP-RR protocol route update method sends route update packets to GW via new BS and ending at cross MRA while in HAWAII protocol route update packets were sent to the old BS via the new BS. For the latter may cause nonoptimal routing, therefore MIP-RR protocol path update method is more worth to recommend.

\section{Conclusion}

This paper makes a study of mobility support protocols network layer handoff delay and analysis focused on the route update time of mobility support protocols. The result shows that route update time is relate to the route update methods of mobility support protocols. Micromobility protocols route update packets were sent only within administration domain, so the route update time is far less than that of Mobile IP protocol. As to different micromobility protocols, CIP protocol route update packets should be sent to GW continuously; route update time is larger. HAWAII protocol route update packets were sent to the old BS directly; route update time was greatly influenced by network structure. The route update method of MIP-RR protocol has an optimal performance, because the route update packets were sent to the GW direction and ended at the cross MRA. MIP-RR represents the development direction of micromobility protocols.

\section{Conflict of Interests}

The authors declare that there is no conflict of interests regarding the publication of this paper.

\section{Acknowledgments}

This work was supported by the Prospective Research Project on Future Networks of Jiangsu Future Networks Innovation Institute (no. BY2013095-1-16) and the National Key Technology R\&D Program of China (no. 2013BAK06B03).

\section{References}

[1] A. Myles, D. B. Johnson, and C. Perkins, "A mobile host protocol supporting route optimization and authentication," IEEE Journal on Selected Areas in Communications, vol. 13, no. 5, pp. 839-849, 1995.

[2] C. Perkins, "IP mobility support for IPv4, revised," Tech. Rep. IETF RFC5944, 2010.

[3] C. Perkins, D. Johnson, and J. Arkko, "Mobility Support in IPv6," IETF RFC6275, 2011.

[4] E. Fogelstroem, A. Jonsson, and C. Perkins, "Mobile IPv4 regional registration," IETF RFC 4857, Internet Engineering Task Force, Dallas, Tex, USA, 2007.

[5] I.-R. Chen, W. He, and B. Gu, "Proxy-based regional registration for integrated mobility and service management in mobile IP systems," Computer Journal, vol. 50, no. 3, pp. 281-293, 2007.

[6] S. Omer, S. Qamar, R. Vesilo, and E. Dutkiewicz, "Efficient mobility management using simplified cellular IP," in Proceedings of the Australasian Telecommunication Networks and Applications Conference (ATNAC '13), pp. 13-18, November 2013.

[7] I. V. Hernández and Q. E. E. Morones, "Performance analysis of the cellular IP mobility protocol," IEEE Latin America Transactions, vol. 5, no. 2, pp. 99-102, 2007.

[8] R. Ramjee, K. Varadhan, L. Salgarelli, S. R. Thuel, S.-Y. Wang, and T. La Porta, "HAWAII: a domain-based approach for supporting mobility in wide-area wireless networks," IEEE/ACM Transactions on Networking, vol. 10, no. 3, pp. 396-410, 2002.

[9] A. Wali, M. A. Ghazali, and S. R. Ayyubi, "Comparative analysis of mobile IP and HAWAII," Communications in Computer and Information Science, vol. 20, pp. 169-179, 2008.

[10] A. T. Campbell, J. Gomez, S. Kim, C.-Y. Wan, Z. R. Turanyi, and A. G. Valko, "Comparison of IP micromobility protocols," IEEE Wireless Communications, vol. 9, no. 1, pp. 72-82, 2002.

[11] K. Pei, J.-D. Li, and F. Guo, "Mobile IP routing optimization performance analysis and simulation," Journal of Electronics, vol. 30, 2002.

[12] T. Hong, X. S. Min, W. Z. Fu, and Z. Jun, "Improved mobile IP register performance analysis," Journal of Communication, vol. 26, no. 6, 2002.

[13] A.-Q. Zhao, "Investigation on handoff performance of mobility support protocols," Journal of Software, vol. 16, no. 4, pp. 587594, 2005.

[14] L. Piroska, A. M. Ronai, and R. Z. Turanyi, "Cost of location maintenance related signaling in IP micro mobility networks," in Proceedings of the Transcom Conference, Zilina, Slovakia, June 2001.

[15] T. Pagtzis and C. Perkins, "Performance issues for localized IP mobility management," in Proceedings of the IEEE International Conference on Networks (ICON '02), August 2002.

[16] J.-H. Lee, T. Ernst, and T.-M. Chung, "Cost analysis of IP mobility management protocols for consumer mobile devices," IEEE Transactions on Consumer Electronics, vol. 56, no. 2, pp. 1010-1017, 2010.

[17] M. Song and J. Jeong, "Performance analysis of a novel internetworking architecture for cost-effective mobility management support," KSII Transactions on Internet and Information Systems, vol. 8, no. 4, pp. 1344-1367, 2014.

[18] A.-Q. Zhao, "Research on scalability of mobility support protocols," Journal of Harbin Institute of Technology, vol. 38, no. 10, pp. 1732-1735, 2006. 

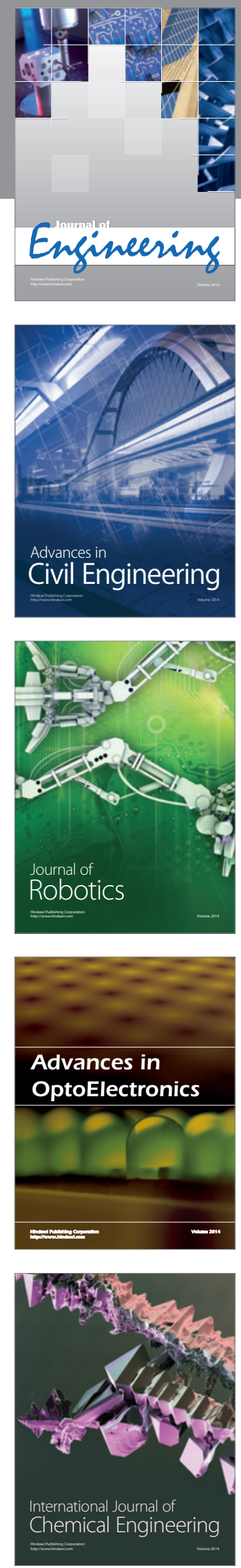

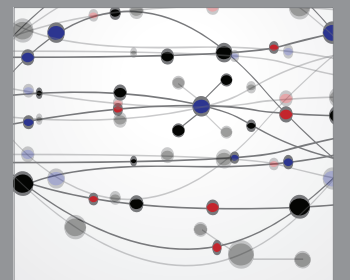

The Scientific World Journal
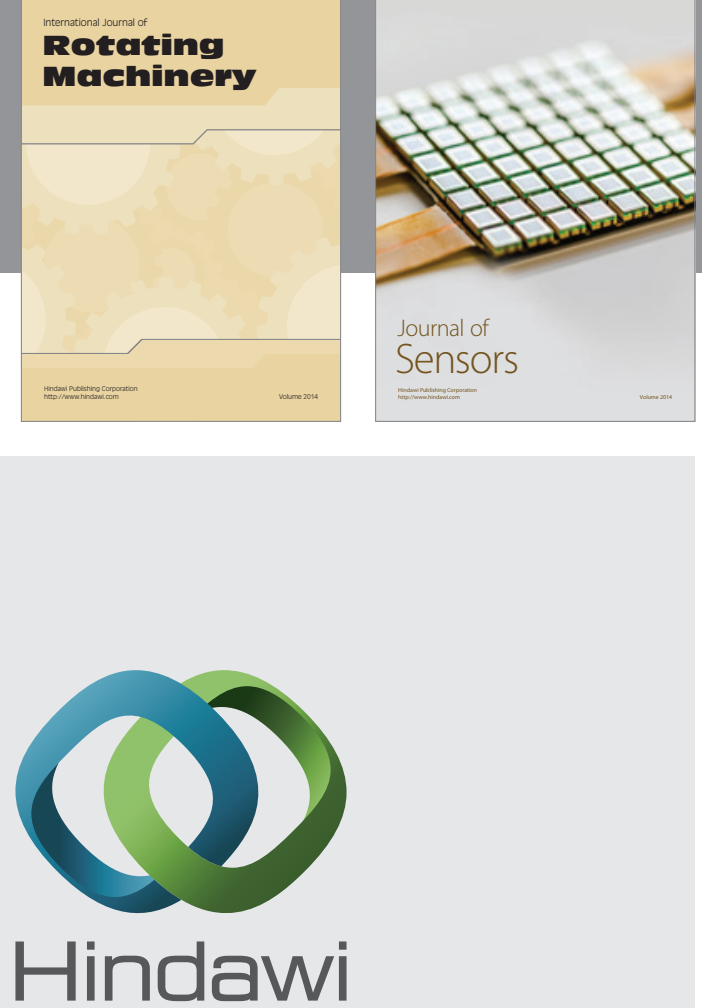

Submit your manuscripts at http://www.hindawi.com
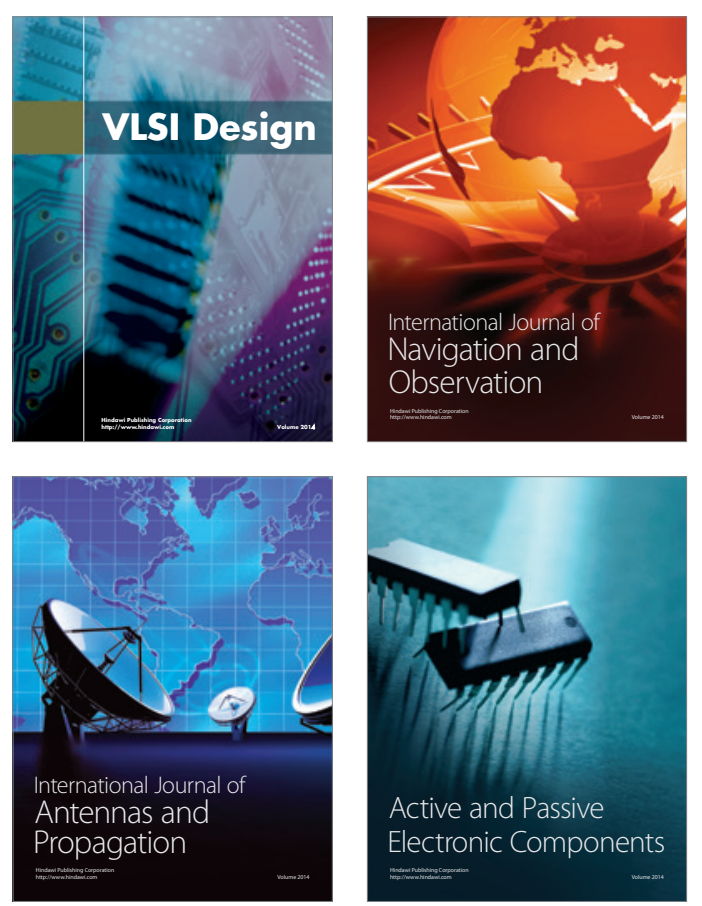
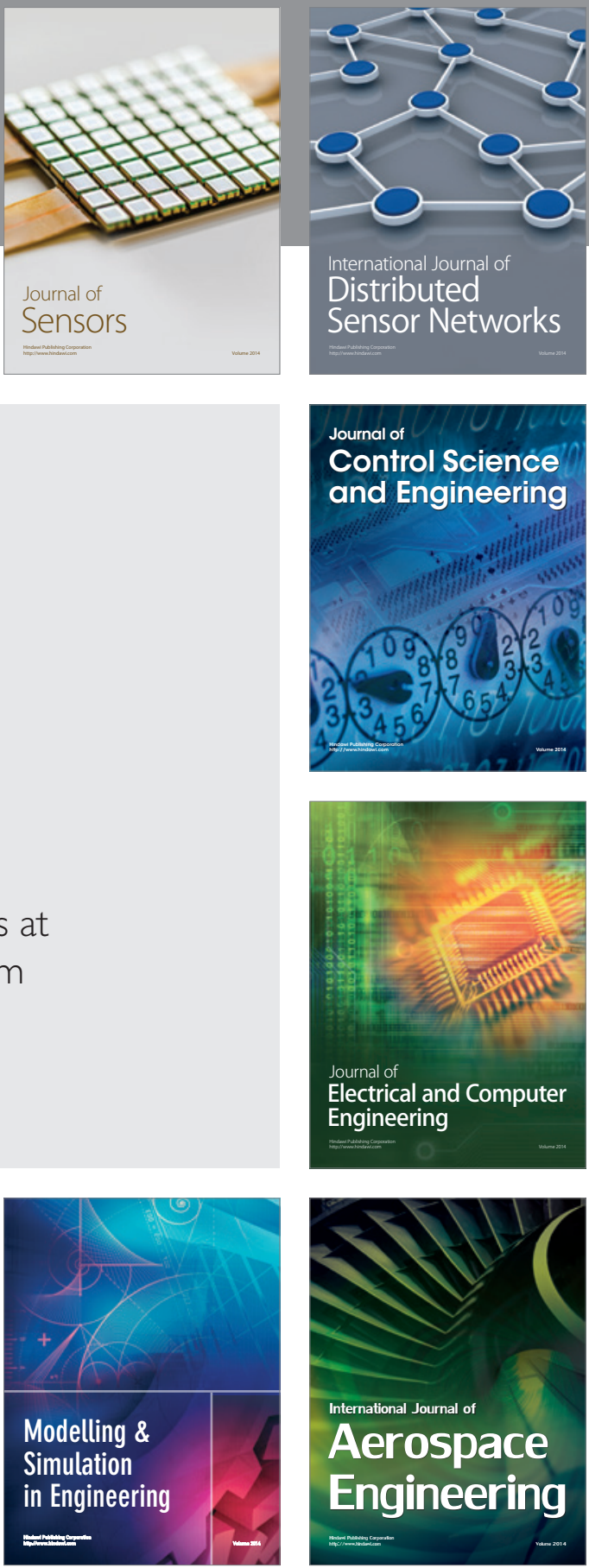

Journal of

Control Science

and Engineering
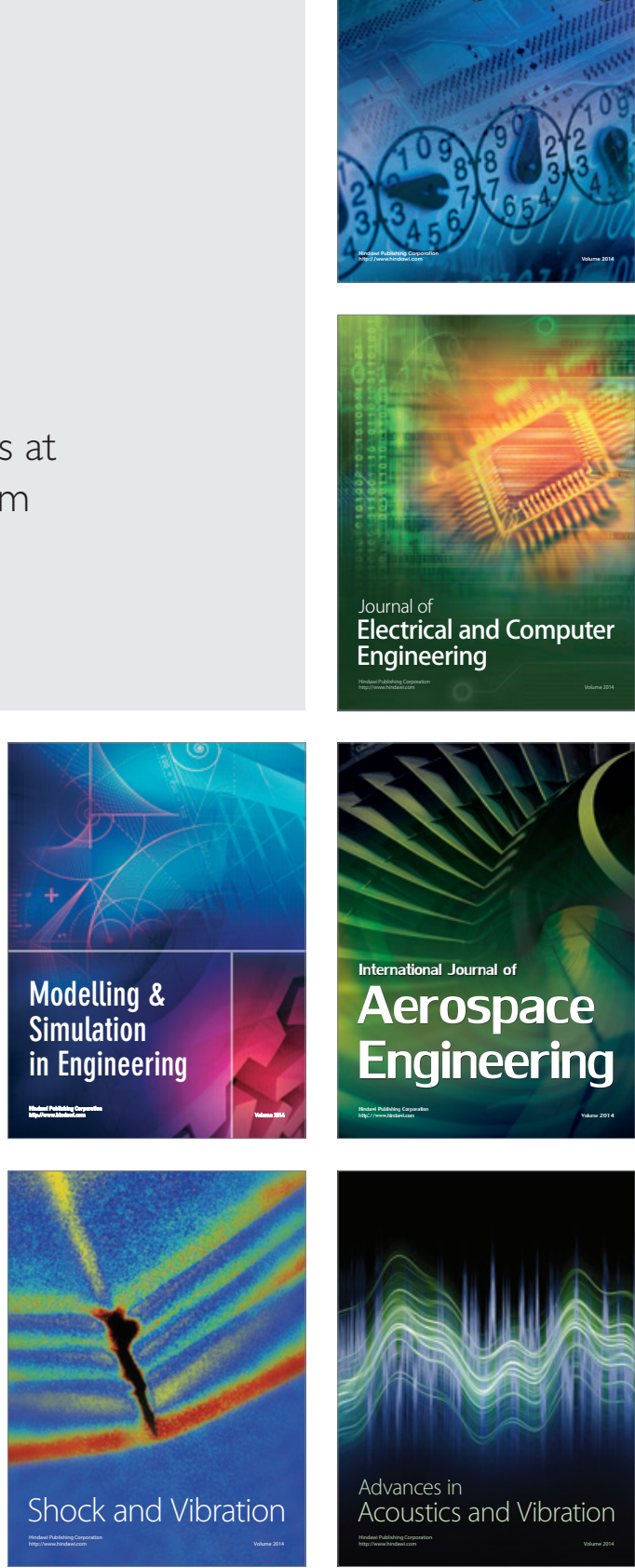